\title{
Prognosis for Patients With Traumatic Cervical Spinal Cord Injury Combined With Cervical Radiculopathy
}

\author{
Seo Yeon Kim, MD¹, Tae Uk Kim, MD¹, Seong Jae Lee, MD, PhD², Jung Keun Hyun, MD, PhD ${ }^{1,3,4}$
}

\begin{abstract}
${ }^{1}$ Department of Rehabilitation Medicine, Dankook University College of Medicine, Cheonan; ${ }^{2}$ Department of Rehabilitation Medicine, National Rehabilitation Center, Seoul; ${ }^{3}$ Department of Nanobiomedical Science, BK21 PLUS NBM Global Research Center for Regenerative Medicine, Dankook University, Cheonan; ${ }^{4}$ Institute of Tissue Regeneration Engineering, Dankook University, Cheonan, Korea
\end{abstract}

Objective To delineate cervical radiculopathy that is found in combination with traumatic cervical spinal cord injury (SCI) and to determine whether attendant cervical radiculopathy affects the prognosis and functional outcome for SCI patients.

Methods A total of 66 patients diagnosed with traumatic cervical SCI were selected for neurological assessment (using the International Standards for the Neurological Classification of Spinal Cord Injury [ISNCSCI]) and functional evaluation (based on the Korean version Modified Barthel Index [K-MBI] and Functional Independence Measure [FIM]) at admission and upon discharge. All of the subjects received a preliminary electrophysiological assessment, according to which they were divided into two groups as follows: those with cervical radiculopathy (the SCI/Rad group) and those without (the SCI group).

Results A total of 32 patients with cervical SCI (48.5\%) had cervical radiculopathy. The initial ISNCSCI scores for sensory and motor, K-MBI, and total FIM did not significantly differ between the SCI group and the SCI/Rad group. However, at discharge, the ISNCSCI scores for motor, K-MBI, and FIM of the SCI/Rad group showed less improvement $(5.44 \pm 8.08,15.19 \pm 19.39$ and $10.84 \pm 11.49$, respectively) than those of the SCI group (10.76 \pm 9.86 , $24.79 \pm 19.65$ and $17.76 \pm 15.84$, respectively) $(\mathrm{p}<0.05)$. In the SCI/Rad group, the number of involved levels of cervical radiculopathy was negatively correlated with the initial and follow-up motors score by ISNCSCI.

Conclusion Cervical radiculopathy is not rare in patients with traumatic cervical SCI, and it can impede neurological and functional improvement. Therefore, detection of combined cervical radiculopathy by electrophysiological assessment is essential for accurate prognosis of cervical SCI patients in the rehabilitation unit.

Keywords Spinal cord injuries, Radiculopathy, Prognosis, Electrophysiology, Patient outcome assessment

Received May 13, 2014; Accepted June 5, 2014

Corresponding author: Jung Keun Hyun

Department of Rehabilitation Medicine, Dankook University College of Medicine, 119 Dandae-ro, Dongnam-gu, Cheonan 330-997, Korea

Tel: +82-41-550-3889, Fax: +82-41-551-7062, E-mail: rhhyun@dankook.ac.kr

() This is an open-access article distributed under the terms of the Creative Commons Attribution Non-Commercial License (http://creativecommons.org/ licenses/by-nc/3.0) which permits unrestricted noncommercial use, distribution, and reproduction in any medium, provided the original work is properly cited. Copyright ( 2014 by Korean Academy of Rehabilitation Medicine 


\section{INTRODUCTION}

Spinal cord injury (SCI) is one of the major and potentially devastating lesions, because spontaneous restoration is rare and there is still no completely effective treatment to date [1]. In the rehabilitation setting, prediction of SCI patients' functional outcome based on their neurological status is integral to formulations of rehabilitation goals and determinations of the lengths of hospital stays. The clinical factors affecting SCI prognosis include age, medical comorbidity, and spasticity, and in the cases of incomplete SCI, even education status can be a clinical factor [2,3]. Combined peripheral nerve injuries and anterior horn cell or root damage, detectable by electrophysiological assessment, also can influence functional outcomes for SCI patients [4].

With respect to traumatic cervical SCI, there has been no report on peripheral nerve or root injury incidence or prognosis. There have been a few reports on combined brachial plexus injury (BPI) in the cases of cervical SCI [5-7] or other trauma of spine, although they are relatively considered to be extremely rare [8]. Because the combined cervical root injury can interfere with SCI patients' recovery, its detection is essential to the determination of functional goals in the rehabilitation setting. The purpose of the present study was to delineate instances of combined cervical radiculopathy in patients with traumatic cervical SCI and to determine whether combined cervical radiculopathy affects prognosis and functional outcome in such cases.

\section{MATERIALS AND METHODS}

\section{Subjects}

A total of 66 patients with traumatic cervical SCI confirmed by magnetic resonance imaging were evaluated in this study. All of the patients had a history of cervical spine fracture or disc herniation. Any potential subjects with the following conditions had been excluded: preexisting or combined brain lesions, systemic polyneuropathy, other peripheral neuropathy affecting electrophysiological abnormalities, musculoskeletal disorders that would affect the accuracy of neurological or functional assessment, and spinal-shock stage within seven days of injury. All of the included subjects underwent neurological, functional, and electrophysiological assessments at the admission to the rehabilitation unit. According to the electrophysiological findings, the subjects were divided to two groups as follows: SCI patients with cervical radiculopathy (the SCI/Rad group) and those without (the SCI group). This study was approved by the Institutional Review Board of Dankook University.

\section{Methods}

Neurological assessment

All of the subjects underwent neurological assessment according to the International Standards for the Neurological Classification of Spinal Cord Injury (ISNCSCI; revised by the American Spine Injury Association [ASIA] in 2011 [9]) at the admission and the follow-up at more than four weeks from the initial assessment. Severity of SCI was evaluated on the ASIA impairment scale (AIS). In comparing the clinical findings between the SCI/Rad and SCI groups, we also evaluated the motor scores and sensory scores along with the total motor and sensory scores for the C5-T1 vertebrae.

\section{Electrophysiological assessment}

The electrophysiological assessment was performed no sooner than three weeks following SCI using a Medelec Synergy TECA machine (Oxford Instruments, Oxfordshire, UK). The electrophysiological criteria for cervical radiculopathy were as follows: detection of abnormal spontaneous activities in cervical paraspinal muscles and in C5-T1 myotomes at rest [10], with normal latency of median and ulnar sensory nerve action potentials in the sensory nerve conduction study; and normal latency of median and ulnar compound muscle action potential in the motor nerve conduction study. For the detection of abnormal spontaneous activities in cervical paraspinal muscles, concentric needle electrode was inserted 1-2 $\mathrm{cm}$ lateral to the cervical spinous process, as described previously [11]. Additionally, the somatosensory evoked potentials were determined by mixed nerve (median, ulnar, peroneal, and tibial) stimulation and C5-T1 dermatomal stimulation.

\section{Functional assessment}

The subjects were assessed according to the Korean version of Modified Barthel Index (K-MBI) and Functional Independence Measure (FIM) at the admission and upon follow-up at more than four weeks after the initial assessment. The K-MBI was evaluated according to the standard protocol (10-item scale, $0-100$ score) for 
feeding, grooming, dressing, bed and wheelchair mobility, transference to and from a toilet, bathing, walking on a level surface, going up and down the stairs, and bladder and bowel continence. FIM also was evaluated according to the standard protocol (18-item scale, 1-7 score), specifically to assess the level of independence (total score range, 18-126) in relation to self-care, sphincter control, mobility, communication, psychosocial state, and cognition.

\section{Statistics}

A statistical analysis was performed using PASW Statistics ver. 18 for Windows (IBM, Armonk, NY, USA). Fisher exact test was used to analyze the baseline categorical data for gender and the AIS results at admission. The Mann-Whitney U test was used to compare the SCI and SCI/Rad groups' numerical data by age, evaluation period, motor score, sensory score, and functional parameters. The Wilcoxon signed-rank test was utilized to compare the changes in motor score, sensory score, and functional parameters from admission to follow-up. A Spearman rank correlation analysis was performed to delineate the relationships among clinical, neurological, and functional results. The statistical significance was set at $\mathrm{p}<0.05$.

\section{RESULTS}

Among the 66 SCI patients, 32 (48.5\%) had cervical radiculopathy (Table 1). The gender and age distributions did not differ between the SCI/Rad and SCI groups. At the admission, 10 patients in the SCI/Rad group had motor complete injury (AIS A and B, 31.2\%), compared to 7 patients in the SCI group (20.6\%). The AIS distribution (from A to D) did not differ between the groups (Table 1).

While the patients with motor incomplete injury showed neurological improvements in total and upper limbs (C5-T1) sensory and motor scores, those with motor complete injury in either the SCI or SCI/Rad group did not show any improvement until the follow-up (Table 2). The initial ISNCSCI sensory and motor scores of the SCI group (84.47 \pm 31.82 and $64.76 \pm 28.11$, respectively) and SCI/Rad group (75.25 \pm 28.37 and $52.66 \pm 29.11$, respectively) did not differ, and neither did the sensory score improvement, regardless of the completeness of SCI. However, the motor score improvement in patients with motor incomplete injury was higher in the SCI group (12.19 \pm 10.08 and $8.70 \pm 6.97$ for total and upper limbs, respectively) than in the SCI/Rad group (7.09 \pm 9.20 and 5.59 \pm 6.51 , respectively) (Table 2).

The initial K-MBI and total FIM scores did not differ between the SCI group $(34.85 \pm 32.70$ and $70.24 \pm 23.18$, respectively) and the SCI/Rad group (25.81 \pm 26.67 and $62.75 \pm 19.44$, respectively), and all of the subjects showed improvement at follow-up, regardless of the group or completeness of SCI (Table 3). However, when comparing the follow-up K-MBI and total FIM scores between the SCI and SCI/Rad groups, there were higher gains in the SCI group than in the SCI/Rad group (Table 3).

Additionally, we found that the number of levels in-

Table 1. Baseline characteristics of SCI and SCI/Rad groups

\begin{tabular}{lccc}
\hline \multicolumn{1}{c}{ Characteristic } & SCI (n=34) & SCI/Rad (n=32) & p-value \\
\hline Gender (male:female) & $27: 7$ & $29: 3$ & $0.306^{\mathrm{a})}$ \\
Age (yr) & $51.24 \pm 12.67$ & $46.72 \pm 12.23$ & $0.146^{\text {b) }}$ \\
Duration from onset to initial evaluation (day) & $48.29 \pm 87.03$ & $75.19 \pm 122.83$ & $0.306^{\mathrm{b})}$ \\
Duration from initial evaluation to follow-up (day) & $59.82 \pm 49.51$ & $64.63 \pm 39.73$ & $0.667^{\mathrm{b})}$ \\
AIS at admission & & & $0.405^{\mathrm{a})}$ \\
AIS A & $4(11.8)$ & $5(15.6)$ & \\
AIS B & $3(8.8)$ & $5(15.6)$ & \\
AIS C & $5(14.7)$ & $8(25.0)$ & \\
AIS D & $22(64.7)$ & $14(43.8)$ & \\
\hline
\end{tabular}

Values are presented as number (\%) or mean \pm standard deviation.

SCI, patients with cervical spinal cord injury but no cervical radiculopathy; SCI/Rad, patients with cervical spinal cord injury and cervical radiculopathy; AIS, American Spinal Injury Association impairment scale.

${ }^{\text {a) }}$ Fisher exact test, ${ }^{\text {b) }}$ Mann-Whitney U test. 


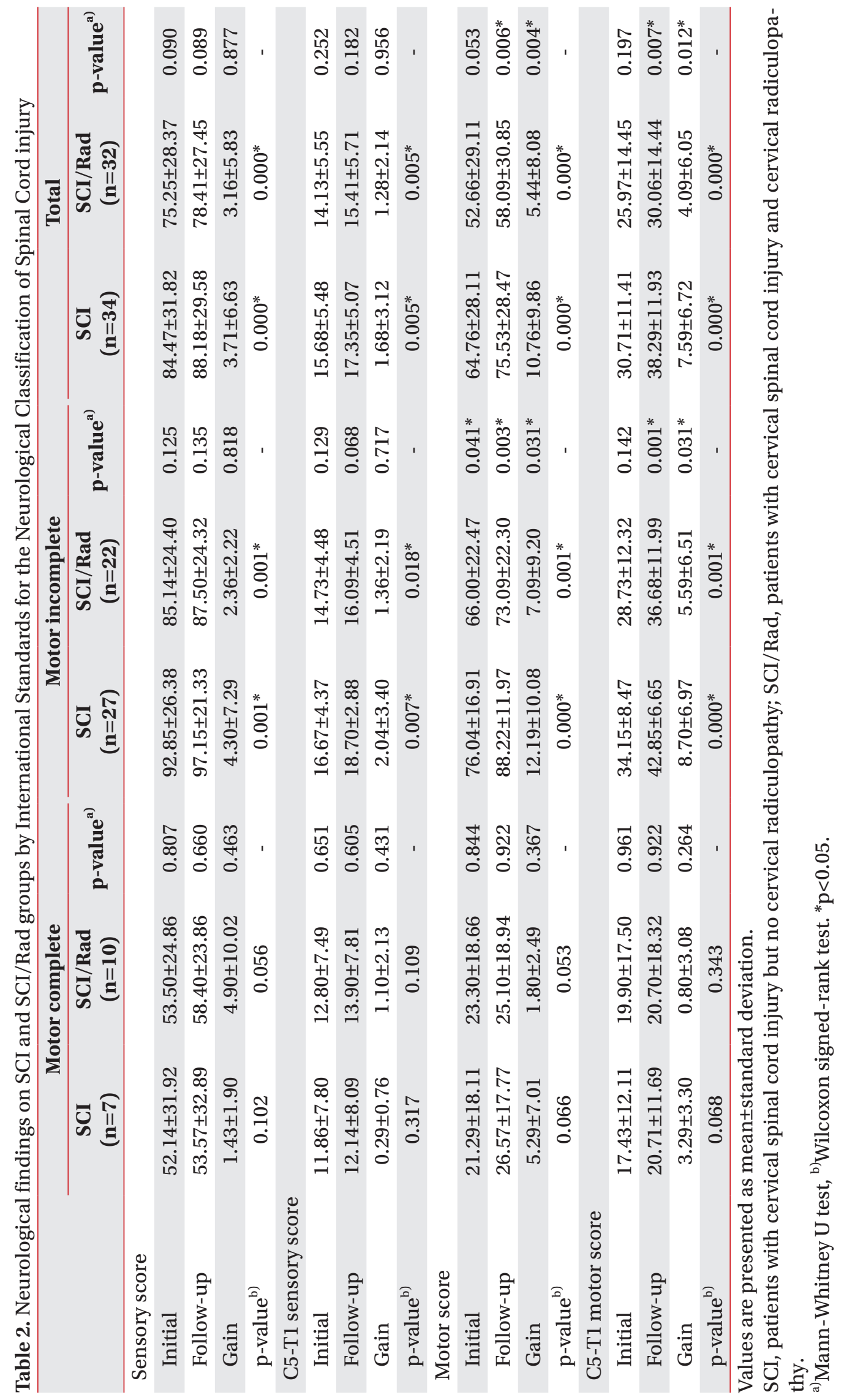




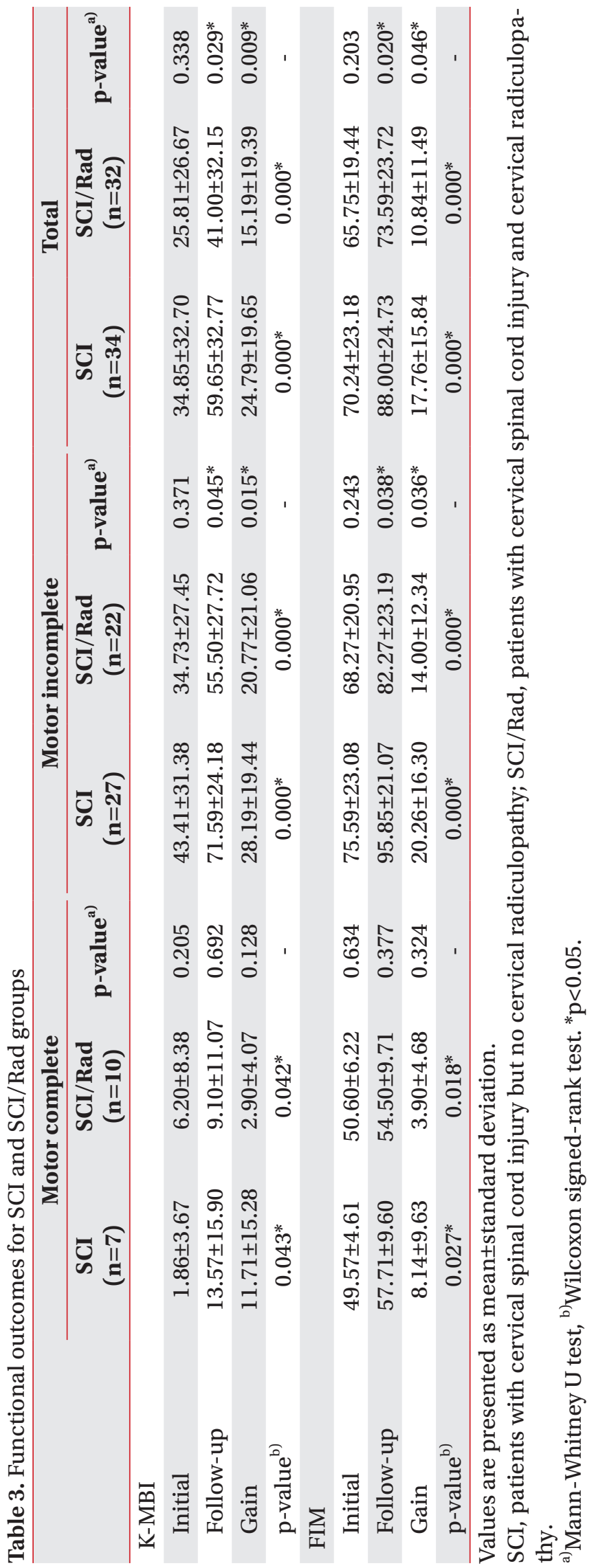

Table 4. Correlation between number of involved root levels and neurological findings in SCI/Rad group

\begin{tabular}{|c|c|c|}
\hline & Coefficient & p-value ${ }^{a)}$ \\
\hline \multicolumn{3}{|l|}{ Motor score } \\
\hline Initial & -0.484 & $0.005^{*}$ \\
\hline Follow-up & -0.396 & $0.025^{*}$ \\
\hline \multicolumn{3}{|c|}{ C5-T1 motor score } \\
\hline Initial & -0.470 & $0.007^{*}$ \\
\hline Follow-up & -0.392 & $0.027^{*}$ \\
\hline
\end{tabular}

volved in cervical radiculopathy in the SCI/Rad group was negatively correlated with the initial and follow-up motor scores (Table 4).

\section{DISCUSSION}

Predicting functional outcomes in patients with SCI is integral to the establishment of goals in the rehabilitation setting. SCI prognosis relies on the level and severity of injury, which is based on the ISNCSCI assessments. However, little is known about comorbidity factors, such as peripheral nerve injury or whether they affect SCI prognosis. One factor is root avulsion or anterior horn cell damage, which can accompany spinal trauma or SCI. In the present study, we found that almost half of the cervical SCI patients $(48.5 \%$ ) had cervical radiculopathy. In fact, no incidences of combined cervical radiculopathy in SCI patients have been known until now. Some researchers have reported that combined BPI is very rare in patients with SCI $[5,12]$. Indeed, their surgical findings were limited to root avulsion or rupture, which is therefore represented as cases of radiculopathy rather than of BPI.

Completeness of motor impairment (AIS A and B vs. AIS C and D) is an important factor determining recovery from neurological and functional impairments, regardless of the cervical radiculopathy combination. Among our patients with motor complete injury, we found that the sensory and motor as well as the K-MBI and FIM scores were not improved during the follow-up period, in either the SCI or the SCI/Rad group (Tables 2, 3). Fawcett et al. [13] reported that more than $80 \%$ of AIS A cases remained as AIS A until 12 months following SCI, and the rate of spontaneous recovery of AIS B was less than $40 \%$, 
while AIS C and D showed improvement in more than $60 \%$ and $95 \%$ of the cases, respectively.

Similar to the existence of cervical radiculopathy, the number of involved roots was also negatively correlated with motor score at the admission and at the follow-up (Table 4). This root counting method can be an effective alternative means of determining radiculopathy severity, as combined radiculopathy/SCI renders difficulty of precise detection.

Regarding the sensory scores obtained in this study, the SCI and SCI/Rad groups did not show any significant differences. Most of the SCI patients $(30 / 34,88.2 \%)$ and SCI/ Rad patients $(27 / 32,84.3 \%)$ were AIS B to D, and their sensory impairments were incomplete and not prominent. Moreover, the narrow sensory grading system (0-2) of the ISNCSCI might have masked small differences between the SCI and SCI/Rad groups.

Our study has two limitations. First, the follow-up period was not fixed, and this might have affected the SCI and SCI/Rad patients' prognoses. Although we found no statistical correlation between the follow-up period and the clinical and functional results, a two-month (60-65 days) follow-up period is not sufficient for the consideration of combined cervical radiculopathy with a definite prognostic factor for cervical SCI. Motor recovery is usually rapid during the first three months, attaining a plateau at 12-18 months following SCI [13]. Therefore, a follow-up period of more than a year is necessary for more complete elucidation of the difference between SCI and SCI/ Rad groups. The second limitation of this study is that the cervical radiculopathy itself could lower sensory and motor scores, as well as AIS, and even the functional scores. This would make it difficult to distinguish the proportion of central nerve damage from that of peripheral nerve damage, which affects the neurological and functional status of SCI patients. Given the possibility of peripheral nerve regeneration, SCI patients with combined cervical radiculopathy might recover more quickly than patients with only SCI. But in some cases, this could mask the prognosis-worsening effect of the combined peripheral nerve injury in SCI. Harrop et al. [14] found that SCI at the thoracolumbar level, when combined with lower motor neuron lesions, such as cauda equina lesions, showed to be contrary to our result by having better outcomes than SCI at the upper-thoracic level. However, because most of the subjects in that study had mild injury (AIS D), it is possible that SCI itself is not obvious when combined with lower motor neuron lesions.

In this study, we achieved two key findings: first, the cervical radiculopathy is not rare in patients with cervical SCI; and second, it can impede neurological and functional improvement. Therefore, for accurate prognosis of cervical SCI patients in the rehabilitation unit, detection of combined cervical radiculopathy by electrophysiological assessment is essential.

\section{CONFLICT OF INTEREST}

No potential conflict of interest relevant to this article was reported.

\section{ACKNOWLEDGMENTS}

This research was supported by a grant of the Korea Health Technology R\&D Project through the Korea Health Industry Development Institute, funded by the Ministry of Health \& Welfare, Republic of Korea (No. HI14C0522).

\section{REFERENCES}

1. Hyun JK, Kim HW. Clinical and experimental advances in regeneration of spinal cord injury. J Tissue Eng 2010;2010:650857.

2. Dvorak MF, Fisher CG, Hoekema J, Boyd M, Noonan V, Wing PC, et al. Factors predicting motor recovery and functional outcome after traumatic central cord syndrome: a long-term follow-up. Spine (Phila Pa 1976) 2005;30:2303-11.

3. Pollard ME, Apple DF. Factors associated with improved neurologic outcomes in patients with incomplete tetraplegia. Spine (Phila Pa 1976) 2003;28:33-9.

4. Curt A, Dietz V. Electrophysiological recordings in patients with spinal cord injury: significance for predicting outcome. Spinal Cord 1999;37:157-65.

5. Akita S, Wada E, Kawai H. Combined injuries of the brachial plexus and spinal cord. J Bone Joint Surg $\mathrm{Br}$ 2006;88:637-41.

6. Rhee PC, Pirola E, Hebert-Blouin MN, Kircher MF, Spinner RJ, Bishop AT, et al. Concomitant traumatic spinal cord and brachial plexus injuries in adult patients. J Bone Joint Surg Am 2011;93:2271-7.

7. Dubuisson AS, Kline DG. Brachial plexus injury: a 
survey of 100 consecutive cases from a single service. Neurosurgery 2002;51:673-83.

8. Midha R. Epidemiology of brachial plexus injuries in a multitrauma population. Neurosurgery 1997;40:11829.

9. Kirshblum SC, Burns SP, Biering-Sorensen F, Donovan W, Graves DE, Jha A, et al. International Standards for Neurological Classification of Spinal Cord Injury (revised 2011). J Spinal Cord Med 2011;34:535-46.

10. Chae SH, Lee SJ, Kim MS, Kim TU, Hyun JK. Cervical multifidus muscle atrophy in patients with unilateral cervical radiculopathy. J Korean Acad Rehabil Med 2010;34:743-51.

11. Dumitru D, Zwarts MJ. Radiculopathies. In: Dumitru D, Amato AA, Zwarts MJ, editors. Electrodiagnostic medicine. 2nd ed. Philadelphia: Haley \& Belfus; 2002. p. 713-76.

12. Grundy DJ, Silver JR. Combined brachial plexus and spinal cord trauma. Injury 1983;15:57-61.

13. Fawcett JW, Curt A, Steeves JD, Coleman WP, Tuszynski MH, Lammertse D, et al. Guidelines for the conduct of clinical trials for spinal cord injury as developed by the ICCP panel: spontaneous recovery after spinal cord injury and statistical power needed for therapeutic clinical trials. Spinal Cord 2007;45:190-205.

14. Harrop JS, Naroji S, Maltenfort MG, Ratliff JK, Tjoumakaris SI, Frank B, et al. Neurologic improvement after thoracic, thoracolumbar, and lumbar spinal cord (conus medullaris) injuries. Spine (Phila Pa 1976) 2011;36:21-5. 